\title{
Virtual Fixtures for Secondary Tasks
}

\author{
G. Lefemine ${ }^{1}$, G. Pedrini ${ }^{2}$, C. Secchi ${ }^{3}$, F. Tesauri ${ }^{4}$, and S. Marzani ${ }^{5}$ \\ ${ }^{1}$ DISMI, University of Modena and Reggio Emilia, via Amendola 2, Morselli \\ Building, 42100 Reggio Emilia, Italy, lefemine.gianvito.34608@unimore.it \\ ${ }^{2}$ DISMI, University of Modena and Reggio Emilia, via Amendola 2, Morselli \\ Building, 42100 Reggio Emilia, Italy, pedrini.guido.39843 \\ ${ }^{3}$ DISMI, University of Modena and Reggio Emilia, via Amendola 2, Morselli \\ Building, 42100 Reggio Emilia, Italy, cristian.secchi@unimore.it \\ ${ }^{4}$ DISMI, University of Modena and Reggio Emilia, via Amendola 2, Morselli \\ Building, 42100 Reggio Emilia, Italy, francesco.tesauri @ unimore.it \\ ${ }^{5}$ DISMI, University of Modena and Reggio Emilia, via Amendola 2, Morselli \\ Building, 42100 Reggio Emilia, Italy, stefano.marzani@ unimore.it
}

\begin{abstract}
The insertion of data in personal devices (e.g. mobile phones, GPS devices) tends to distract us from the primary task (e.g driving) that we are executing because of the necessity of deviating our visual attention to a secondary task. In this work we have tested the benefits introduced by the haptic feedback as a facility for a very common secondary tasks, namely the insertion of strings in an input device. Experiments demonstrate that the presence of virtual fixtures improves performances during input tasks and decreases the distraction of the user from the primary task.
\end{abstract}

Keywords: haptic, virtual fixtures, affordances, secondary tasks, input task

\section{Introduction}

In our daily life, we are surrounded by personal devices (e.g. mobile phones, pocket PCs, GPS) that require our attention both for delivering their outputs and for giving them some inputs (e.g. writing an SMS, inserting a destination in a GPS device). Usually, what we are doing with these personal devices is not our main activity and, therefore, these requests of attention increase the amount of distraction from our primary activity. For example, distraction is a relevant concern about in-vehicle information systems (IVIS): drivers must divert part of their attentive resources from the driving task (primary task), in order to perform input actions and to receive and understand the system output (secondary task), see (Young, 2003). So far, several researches have investigated driver's distraction in order to isolate factors affecting driving performance and to develop

Please use the following format when citing this chapter:

Lefemine, G., et al., 2008, in IFIP International Federation for Information Processing, Volume 272; Human-Computer Interaction Symposium; Peter Forbrig, Fabio Paternò, Annelise Mark Pejtersen; (Boston: Springer), pp. 67-81. 
distraction-mitigating IVIS, see for example (Donmez, 2004). Still, most of these studies mainly addressed to the second half of the problem, namely how the system informative output should be delivered to the driver in order to minimize the distraction impact. Only few studies (e.g. Nowakowski, 2000) have addressed the problem of defining which input strategies for the secondary task could fit the driving context at best, that is, which kind of device could allow users to safely perform input tasks while driving. At present haptic technologies seem to be the most promising way to achieve the result of minimizing distraction on a secondary input task in a driving context. Haptic feedback can be exploited to give to the input device a higher affordance (Gibson, 1977) and, consequently, to make its use easier on behalf of the driver. A first attempt in this direction has been done by BMW, as reported in (Bengler, 2002). The increase of distraction from the primary task is due to the fact that the same sensorial channel (e.g. vision in the driving context) is significantly required for the completion both of the primary (e.g. driving) and of the secondary task (e.g. inserting a destination on a GPS device). In case two different sensorial channels are involved for the completion of the primary and of the secondary tasks, the distraction from the primary task and the completion time of the secondary task should decrease. Very often, the sensorial channel requested for completing a primary tasks is vision. The goal of this work is to evaluate the benefits of the use of the haptic feedback as the main sensorial channel involved in the insertion of data in input devices. We have developed a prototype of a virtual keyboard input system over which two input strategies have been tested and compared. The first strategy basically consists of a virtual keyboard over which the user moves a pointer; each letter is selected by taking the pointer over it and clicking; the visual attention of the user is required for the insertion process. The second strategy endows the keyboard with a set of virtual fixtures (see, for example, Rosenberg, 1993; Bettini, 2004; Payandeh, 2002; Nolin, 2003) which are activated following a search algorithm called SAPETS (Search Algorithm for Possible Endings of Typed Symbols). The goal of SAPETS is to activate, depending on the letters already selected and on a set of words contained in a database, a set of fixtures that suggest to the user the possible completions of the word he/she is introducing. The logic behind this strategy is basically that of reducing the load on the users' visual attention. This mainly happens in two ways: on the one hand, visual scanning among keys is reduced by presenting visual cues; on the other hand, movements to be performed are haptically guided, thus minimizing the need for fine adjustment. Several experiments have been conducted in order to assess whether the presence of virtual fixtures provides a significant benefit for the user both in case the input task is the only one to perform and in case the user has to draw attention to a primary task, being the input task a secondary task. The paper is organized as follows: in Sec. 2 we provide a description of the experimental setup and of the SAPETS search algorithm used for activating the fixtures over the virtual keyboard. In Sec. 3 and Sec. 4 we provide the results of two sets of experiments 
conducted for evaluating the benefits introduced by the virtual fixtures. In the first case, the only task that has to be carried on by the user is the introduction of words through the input device. In the second case the user has to pay attention to a primary task and to insert words through the virtual keyboard. Finally, in Sec. 5, some concluding remarks are reported and some future work is addressed.

\section{The Experimental Setup}

The aim of this section is to describe the prototype of the input interface that has been developed and the algorithm that is used for enabling the virtual fixtures over the keyboard. The prototype is made up of an haptic device through which the user can interact with a virtual environment representing the input device. We have used a Phantom Omni haptic device by Sensable Technologies and we have implemented the virtual input device in a Matlab/Simulink environment using the Virtual Reality Toolbox. The interconnection between the Phantom Omni and the virtual environment has been implemented by using the Handshake Prosense Virtual Touch toolbox. In this way, the overall application can be developed using Simulink. In fact it is possible to interact both with the virtual environment and with the haptic device by means of Simulink blocks and the development and the maintenance of the overall interface is very quick and intuitive. The virtual environment that has been designed represents a QWERTY keyboard, see Figure 1.

The virtual keyboard enables typing of characters strings and has several typing functions and options (e.g. confirm a string, cancel a character). Each key is represented by the correspondent letter and it is enclosed neither in a visual nor in a physical container (unlike the physical keys of the normal keyboards). The user moves, through the Phantom, a pointer that is used for interacting with the keyboard. The selection of a key is made by moving the pointer on the desired character and by pressing the white button that is placed on the body of the stylus. Since the virtual keyboard doesn't have shaped keys, a haptic elastic potential well has been implemented to make keys selection easier: when the cursor is close to the letter (within a circle with radius of $10 \mathrm{~mm}$ and centered in the center of the rectangle in which the letter can be contained), it is elastically attracted to the center. 


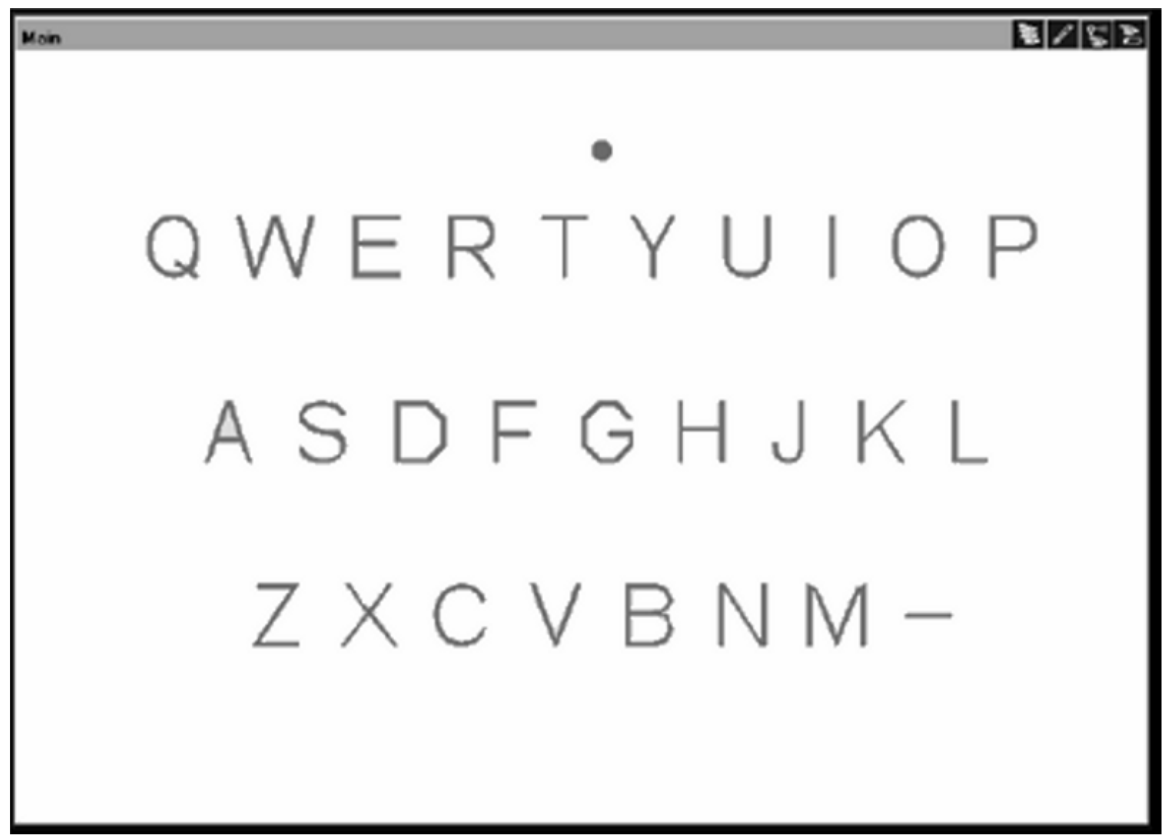

Figure 1 The virtual keyboard layout

In this way, the user can just roughly approach the pointer to the letter and, then, the potential well will take care of bringing the pointer exactly in correspondence of the letter. The user can select the letter over which the cursor is placed by pressing the white button on the stylus. In order to change letter, it is necessary to apply a little force for defeating the elastic force imposed by the potential well around the selected letter and to move the pointer to the next desired point. In this version of the keyboard no haptic facility for inserting words has been introduced. A second virtual keyboard, obtained by endowing the one just described with virtual fixtures for helping the user during the word insertion process, has been developed. For this application, a virtual fixture is a track that joins a pair of letters of the virtual keyboard. When the user moves the pointer along the track, a virtual force constrains the user to keep on moving the pointer along the track. The virtual force is local and, therefore, the user can take the pointer in an off-track position by applying a force that is sufficiently high. The virtual fixtures activation is governed by the SAPETS (Search Algorithm for Possible Endings of Typed Symbols) algorithm. Loosely speaking, the main idea behind this algorithm is to activate the virtual fixtures, namely some preferred directions the user should drive the pointer along, similarly to how the T9 software, developed by Tegic Communications Inc. and present on the most part of mobile phones currently on the market, suggests possible completions of the 
words while typing SMS. A set of words is initially stored in a database. Each time that the user inserts a letter, the SAPETS algorithm is activated. It consists of two stages: the first one searches for all the words that may possibly complete the typing, while the second one looks for all the possible letters that may follow the typed characters. As a result of the SAPETS algorithm, in the second version of the keyboard, after the insertion of each character, visual tracks appears, joining the character to those which could possibly follow it, according to the vocabulary stored in the database; around such tracks is implemented an elastic potential well whose role is to keep the pointer on the track (as proposed in Nolin, 2003; Secchi, 2006). The user can force the pointer to go off the track by applying the amount of force necessary for escaping from the potential well. The virtual fixtures have been placed on a geometric plane which is layered some millimeters over the keyboard plan, thus avoiding conflicts between the tracks layer and the potential wells placed around each letter. That is, if the user is following a virtual fixture and he/she drags the cursor across a key, the elastic attraction effect around the key is not felt. When the user ends composing a word, he/she must press the white button present on the stylus of the Phantom to confirm the selection. It can happen that, especially for some commonly used characters, many completions are possible and, that, therefore, many virtual fixtures would be displayed by the SAPETS algorithm. We have noticed that this can be very disturbing since the attractive effects of the virtual fixtures tend to induce the user to take the pointer along the wrong track. Thus, we have limited the number of tracks that can be displayed to three. The displayed tracks are chosen on the basis of a statistical criterion: once a letter has been selected only the tracks corresponding to the completions of the three words more frequently introduced are displayed. 


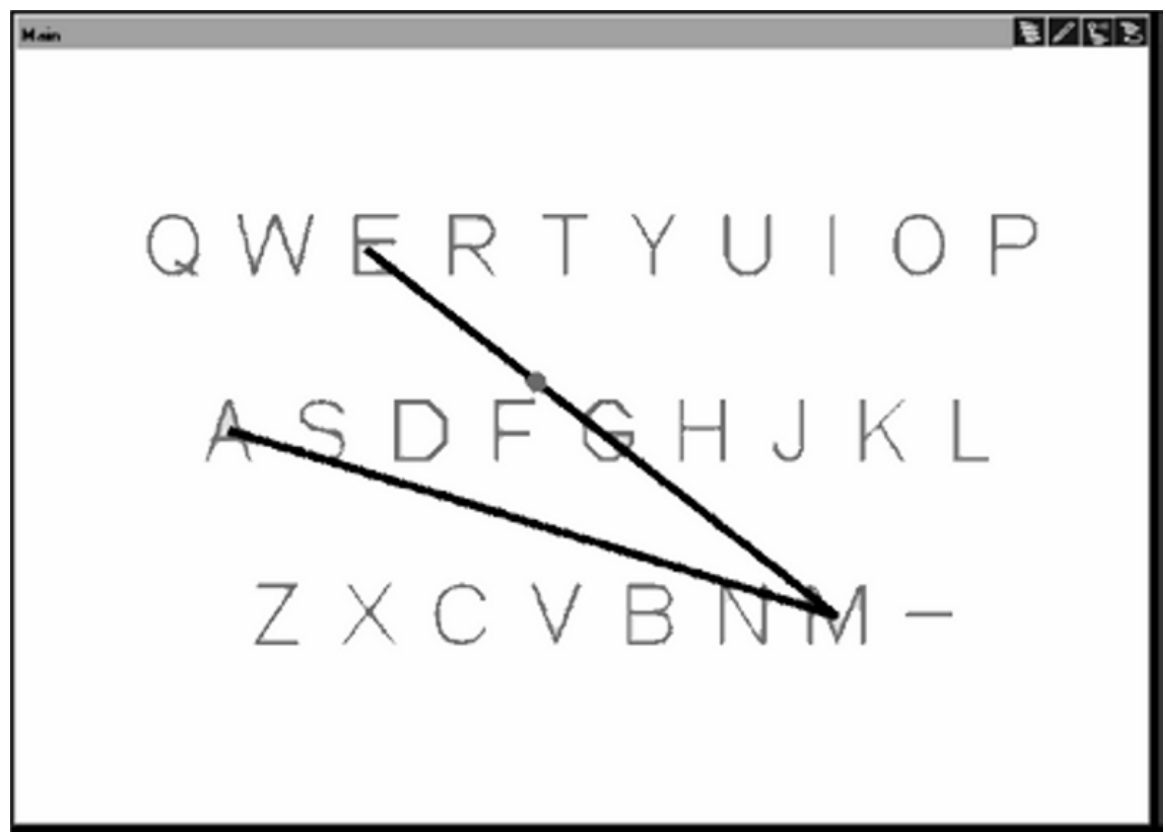

Figure 2 The virtual fixtures displayed using the SAPETS algorithm

To correct typing errors, the right button of a mouse was used. Since the aim of the experiments was to measure the time to type a string we assume that after an error all the typed characters are deleted and the person must restart the insertion process. The virtual keyboard together with some of the virtual fixtures that can be displayed is reported in Figure 2.

\section{Experimental Results in case of single task}

In the first set of experiments we compare the performances obtained by the users in the word insertion process in case the simple virtual keyboard is used with those obtained in case the virtual fixtures activated through the SAPETS algorithm described in Sec. 2 is used. The number of participants to the experiment was twenty four, all participants were right-handed with a mean age of twenty four years in a range from sixteen to forty nine. The user could move the pointer through the Phantom Omni and the virtual keyboard was displayed on a LCD monitor. The participants were divided into two groups, 12 persons each: in the first one, they operated on the first version of the virtual keyboard, namely without any word insertion facility, whereas in the second they could rely on the haptic layer of virtual fixtures described in Sec. 2. Each participant had to compose 24 
words, 12 of which were classified as short ones, namely with less than 5 characters, and 12 as long ones, namely with more than 8 characters. All the words were in Italian, the mother tongue of all participants, since we wanted to avoid errors due to the scarce knowledge of foreign languages. In each group of words, sort ones and long ones, there were 9 common words, whose meaning was well known to all the participants, and 3 uncommon words, whose meaning was unknown by all the users. This choice has been done to simulate what can happen in a driving context, where sometimes the driver could have to insert as a GPS destination a string he/she has never used before (e.g. a small city where he/she has never been before). It was expected that participants would have carried out tasks more rapidly in the condition in which they could rely on virtual fixtures; the benefit deriving from the fixtures was expected to be detected with both short and long words.

Each participant was asked to follow this procedure:

1. read and understand the word

2. place the pointer in a predefined start position

3. when ready to start, push the blue button on the stylus;

4. for each letter of the word the procedure was:

a. place the pointer in correspondence of the character on the keyboard

b. push the white button on the stylus to confirm

c. move to the next position

5. when the word is fully composed, press the blue button again.

After the execution of the test, the participants were asked to fill in a questionnaire presenting 5 questions about the usability of the system where the answers had to be selected on a 7-level Likert scale (Likert, 1932). Each participant was asked to do all actions naturally and without distraction. The movements of the participants' hands and the monitor were recorded during the execution of the experiment since we believe that the clip analysis may lead to the detection of features to improve in possible future developments. Execution times and errors were saved in log files. The use of fixtures (or not) and the word length (long or short) are the variables chosen for the performance analysis. For the group that was not using the virtual fixtures, the average times $T_{\Sigma}^{A F}$ and $F_{i}^{A F}$ for inserting a short word and a long word were respectively:

$$
T_{\Sigma}^{M F}=8.0823 \mathrm{~s} . \quad T_{i}^{M F}=19.3752 \mathrm{~s} .
$$

For the group that was using the virtual fixtures instead, the average times $F_{\mathrm{s}}$ and $F_{i}$ for inserting the short words and the long words were respectively:

$$
T_{E}=8.0823 s . \quad \quad F=19.3752 s .
$$

In Figure 3 we have reported a graphical representation of the average insertion time versus the word length. We can see that the virtual fixtures activated through the SAPETS algorithm introduce a beneficial effect by lowering the average 
insertion time. The benefits of the virtual fixtures become more relevant for long words. The activation of the virtual fixtures helps the user to rapidly move from one letter to the other on the virtual keyboard. The elastic potential well around the fixture helps the user to keep the right track so that he/she can rapidly move towards the desired letter.

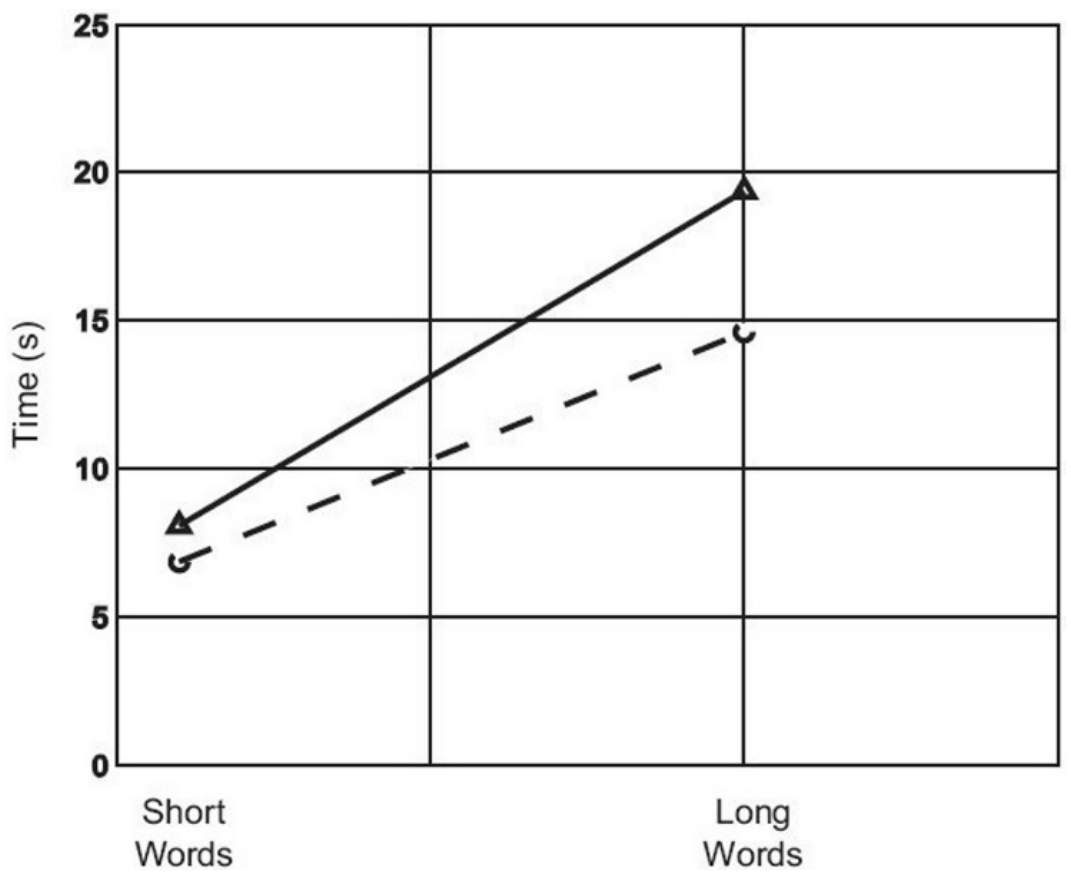

Figure 3 Average time for inserting the words with (dashed) and without (solid) fixtures The virtual fixtures displayed using the SAPETS algorithm

In order to analyze the errors committed by the users during the words insertion task, we have grouped the possible errors into 4 categories:

1. Repeated key typing error: a letter is selected more than once by pushing the button on the stylus.

2. Near key error: a letter near to the desired one is selected

3. Accidental typing error: a wrong letter is selected

4. Distraction error: a letter in the word is not inserted

In Table 1, the number of errors committed by the user during the experiment are reported 
Table 1. Errors Analysis

\begin{tabular}{ccc}
\hline Error Category & With Fixtures & Without Fixtures \\
\hline Repeated key typing & 29 & 9 \\
\hline Near key & 1 & 4 \\
\hline Accidental typing & 4 & 4 \\
\hline Distraction & 4 & 10 \\
\hline
\end{tabular}

The group of participants that use the virtual fixtures usually commits less errors. In particular, near key and distraction errors are significantly less when using fixtures. Thus, it seems that the presence of the virtual fixtures decreases the level of distraction and, consequently, the number of errors. The repeated key typing errors in the group that use fixtures is surprisingly higher than in the other group. Thus, it seems that the presence of the fixtures tends to make the user more unsure about the selection of a letter. Nevertheless, this kind of errors can be easily filtered via software. We aim at experimentally studying this phenomenon more in detail in the future. Once that the benefits of the haptic layer have been tested, it is necessary to evaluate the usability of the interface. In fact, if the users found the input device hard to use and if the system required too much attention for being used, it wouldn't be suitable for being used for the execution of a secondary task since it would tend to distract the user too much. In order to assess the usability of the interface, we have asked to each participant to evaluate, in a scale from 1 (absolutely no) to 7 (absolutely yes) the following statements:

1. generally the system is easy to use;

2. the system helps me to easily complete the assigned task;

3. the system has all the required functions and capacities

4. it is easy understanding when I commit an error;

5. the use of the system is intuitive.

The average evaluation of each statement is collected in Table 2.

Table 2. Interface Evaluation

\begin{tabular}{ccc}
\hline Statement & With Fixtures & Without Fixtures \\
\hline 1 & 5.57 & 5.33 \\
\hline 2 & 5.58 & 5.33 \\
\hline 3 & 5.92 & 4.92 \\
\hline 4 & 5.5 & 5.08 \\
\hline 5 & 5.67 & 5.75 \\
\hline
\end{tabular}

All the statements were given a high assessment; this means that the interface is perceived intuitive and easy to use. It is remarkable that, in case the virtual fixtures are enabled, the assessments of the statements increase or remain comparable with the corresponding ones in case no fixtures are used; this means that the introduction of the haptic word insertion facility is positively perceived in terms of the usability of the interface. In summary, we have experimentally proven that, in case the input task is the only task that the user has to complete, the virtual 
fixtures activated through the SAPETS algorithm lead to an improvement of the performance which seems to grow larger as long as the task becomes more complex (i.e. words become longer). Thus, the presence of the virtual fixtures is beneficial for the usability of the input device. Nevertheless, in the experiments illustrated in this section, the user can see the virtual keyboard and, therefore, he/she can rely both on the haptic and the visual information. What these results cannot tell is whether the above mentioned benefits would persist in case the input task becomes the secondary task and the visual demand for the primary task becomes quite high (e.g. in a driving context). This situation will be analyzed in detail in the next section.

\section{Experimental Results in case of multiple tasks}

The aim of the second experiment is to evaluate whether the virtual fixtures setup keeps on introducing benefits when the word insertion task becomes a secondary task, as it usually happens in a driving context (e.g. the insertion of a destination in a GPS while driving), and most of the visual attention of the user has to be drawn to a primary task. For this experiment, we have developed a graphical application, represented in Figure 4. It consists of one light grey sphere and four dark grey spheres. During the experiment, the light grey sphere is always visualized while the four dark grey spheres appear all together randomly for short periods of time.

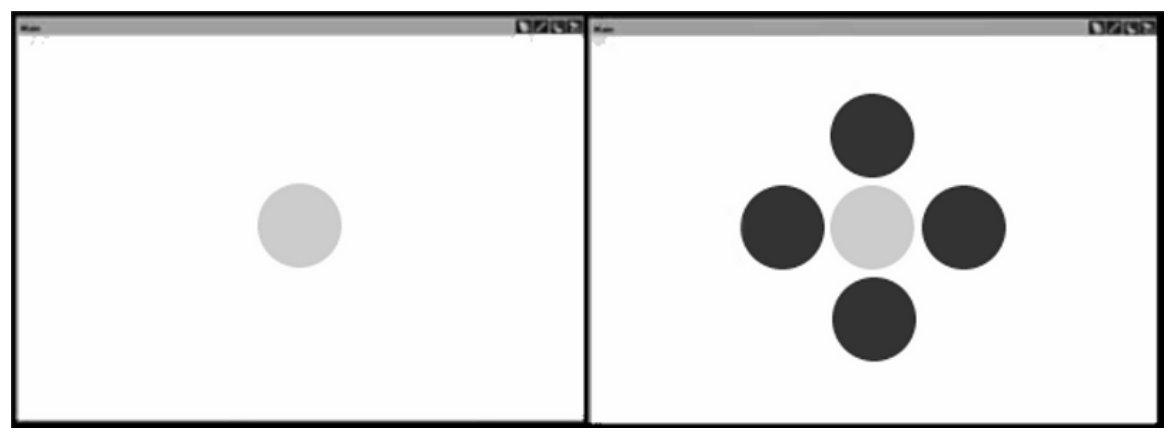

Figure 4 Snapshots of the graphical application that plays the role of the primary task

Each time that the dark grey spheres appear on the screen, the user has to push the space bar. The application allows to count both the number of times that the dark grey spheres appears on the screen and the number of times that the user presses the space bar in correspondence of their appearance. This application provides a simple but meaningful way for implementing a primary task which significantly captures the visual attention of the user. The difference between the number of times that the dark grey spheres have appeared and the number of times that the user has correspondingly pressed the space bar is an indicator of the distraction level of the user. The number of participants to the experiment was 
again twenty four, all participants were right-handed with a mean age of twenty six years in a range from twenty one to fifty eight. Each user was in front of two LCD monitors. The graphical application just described was displayed in one of the monitors and it played the role of the primary task. Each time that the dark grey spheres appeared on the screen, the user had to press the space bar. At the same time, each user was asked to compose 24 words (12 of which were classified as short ones, namely with less than 5 characters, and 12 as long ones, namely with more than 8 characters; all the words were in Italian and 3 words per each group were uncommon) using the virtual keyboard, displayed on the other LCD monitor, together with the Phantom omni as described in Sec. 2. The participants have been divided into two groups of 12 people. A group has used the virtual keyboard without the fixture system while the other one has exploited the virtual fixture facility. The secondary task is a source of distraction from the primary task. We have seen in Sec. 3 that the presence of the virtual fixtures helps the user to insert the words more rapidly. As a result of this experiment, we expected that the users exploiting virtual fixtures would have kept on inserting the words more rapidly than the other users. Furthermore, we expected that the presence of virtual fixtures would have decreased the distraction of the users from the primary task. The participant was asked to follow this procedure:

1. read and understand the first word

2. place the pointer in a predefined start position

3. when ready to start, push the blue button on the stylus; at the same time, the graphical application playing the role of the primary task starts

4. each time that the dark grey spheres appear, press the space bar

5. for each letter of the word the procedure was:

a. place the pointer in correspondence of the character on the keyboard

b. push the white button on the stylus to confirm

c. move to the next position

6. when the word is fully composed, push the blue button again and start over with a new word

After the execution of the test, the participants filled in a questionnaire presenting 5 questions about the usability of the system where the answers had to be selected on a 7-level Likert scale. The participant was asked to do all actions naturally. The movements of the participants' hands and the monitors were recorded during the execution of the experiment. Execution times, the number of times that the user pressed the space bar and the number of times that the dark grey spheres had appeared in the graphical application were saved in log files. Firstly, we aim at assessing whether the use of fixtures keeps on introducing a benefit in the word insertion task also when a primary task is present. As in the previous experiment, the use of fixtures (or not) and the word length (long or short) are the variables chosen for the performance analysis. For the group that was not using the virtual fixtures, the average times $T_{\Sigma}^{W E}$ and $T_{T}^{W E}$ for inserting a short word and a long word were respectively: 


$$
T_{s}^{* F F}=7.7426 s . \quad T_{i} P F=24.1035 \mathrm{~s}
$$

For the group that was using the virtual fixtures instead, the average times $T_{\Sigma}^{F}$ and $T_{i}$ for inserting a short word and a long word were respectively:

$$
T_{\Sigma}^{F}=6.5598 \mathrm{~s} . \quad T_{F}^{F}=19.8226 \mathrm{~s} .
$$

In Figure 5 a graphical representation of the average times versus the length of the words has been reported.

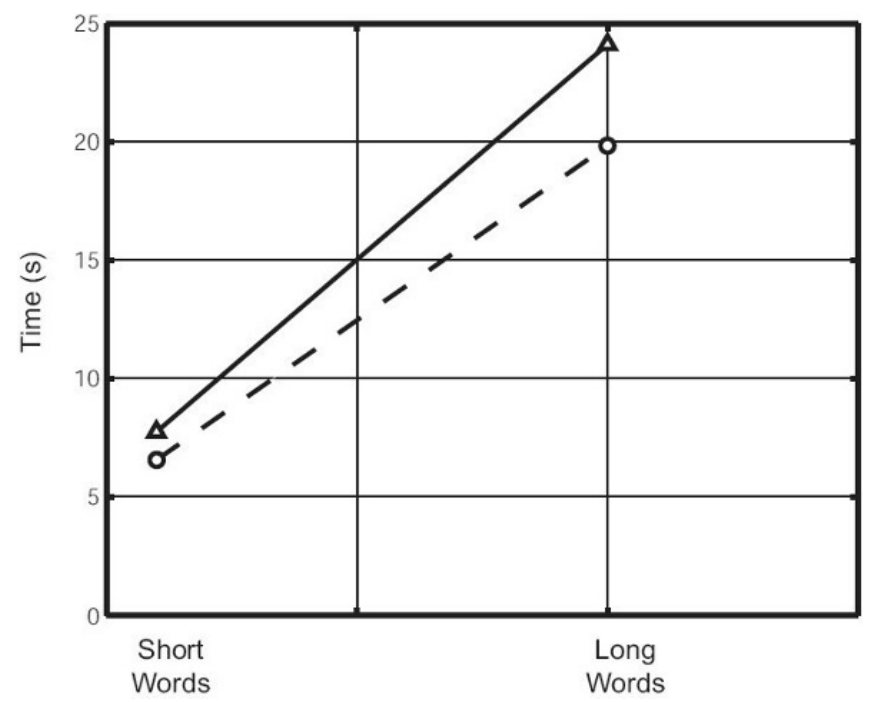

Figure 5 Average time for inserting the words with (dashed) and without (solid) fixtures

We can see that the virtual fixtures keep on introducing a beneficial effect in terms of velocity of insertion. Similarly to the results obtained in Sec. 3, the advantages of the virtual fixtures are more evident in case of the insertion of long words. The presence of the primary task increases the average insertion times with respect to the results obtained in Sec. 3 but the benefits introduced by the virtual fixtures are comparable with those obtained in Sec. 3. Thus, from the comparison of the experiments, it seems that the amount of benefit introduced by the virtual fixtures activated through the SAPETS algorithm is somehow independent of the presence of the primary task. In this second set of experiments we also want to evaluate the influence of the virtual fixtures layer in terms of performances of the primary task. The index used for evaluating the performances of each user on the primary task is the number of times he/she didn't detect the presence of the dark grey spheres because he/she was distracted by the word insertion process, namely by the secondary task. We have counted the total number of errors committed by each group, namely the total number of times that an appearance of the dark grey 
spheres hasn't been detected (i.e. the user didn't press the space bar) during the experiments involving members of the group. For the group that has not used the virtual fixtures the number of errors has been 42 while for the other group it has been 20. This result confirms our expectations: the presence of the virtual fixtures significantly improves performances also in the execution of the primary task. This improvement is mainly due to the fact that the fixtures allow the users to reach the desired letters over the keyboard exploiting mainly the haptic information leaving the user free to devote most of its visual attention to the primary task. In fact, once that the first letter of a word has been entered, the user has just to choose with a glimpse the direction taking the pointer to the next letter and then, thanks to the potential well around the fixture, he/she can let the pointer slide over the virtual track without any need to look at the keyboard. In this way, the time that the user spends at looking to the screen where the primary task is running dramatically increases as well as the number of committed error decreases. On the other hand, without the virtual fixtures, a significant portion of the visual attention of the user has to be devoted to the motion of the pointer over the keyboard and, consequently, he/she misses part of the dark grey spheres appearances. In other words, the presence of virtual fixtures allows to keep the visual attention of the user away from the secondary task which can be executed exploiting almost exclusively the haptic information. Also in this case, we found useful to evaluate the usability of the interface. We have asked to each participant to evaluate, in a scale from 1 (absolutely no) to 7 (absolutely yes) the same statements that have been reported in Sec. 3. The average evaluation of each statement is collected in Table 3.

Table 3. Errors Analysis

\begin{tabular}{ccc}
\hline Statement & With Fixtures & Without Fixtures \\
\hline 1 & 5.25 & 4.46 \\
\hline 2 & 5.92 & 4.61 \\
\hline 3 & 6.5 & 4.5 \\
\hline 4 & 5.76 & 5 \\
\hline 5 & 6.15 & 4.5 \\
\hline
\end{tabular}

Comparing these results with those reported in Table 2, we can see that the evaluations given by the group using the fixtures are, in average, higher. This means that the advantages of the fixtures is perceived by the users also in terms of usability of the interface. On the other hand, without fixtures, the distraction induced by the secondary task is perceived by the users in terms of a decrease of the usability; in fact the evaluations reported in the last column of Table 3 are lower than the corresponding ones in Table 2. In summary, we have experimentally proven that virtual fixtures activated through the SAPETS algorithm improve performance both in terms of velocity of insertion and in terms of errors committed in the primary task. 


\section{Experimental Results in case of multiple tasks}

This paper presents a study on the use of virtual fixtures on input devices for secondary task. We have designed a virtual keyboard and we have proposed an activation algorithm, called SAPETS, for properly activating a set of virtual fixtures. We have conducted experiments that have proven that our algorithm makes the word insertion process faster. Furthermore, in case the input task is secondary and the primary task requires most of the visual attention of the user (e.g. a driving task), our algorithm decreases the distraction from the primary task. Future work aims at developing an input device endowed with virtual fixtures activated by the SAPETS algorithm to be embedded in the in-vehicle information system. Encouraged by the results of this paper, we believe that this input device will decrease the distraction from driving caused by the IVIS. We are building a prototype of a haptic input device that can enable virtual fixtures (see e.g. Wang, 2004) that can be easily integrated in a vehicle. It will be necessary to quantitatively evaluate the benefits of the virtual fixtures in a driving context. We will make experiments using a driving simulator that is being set up in our lab. Specifically, eye-tracking studies will be conducted in a simulated driving environment, in order to collect data regarding the actual visual demand imposed by the secondary task; such data are particularly relevant, since they directly impact on the eyes-off-the-road time, which is critical for drivers' safety.

\section{References}

Bengler, K., Herrler, M. e Künzner, H.: Usability Engineering bei der Entwicklung von iDrive (Usability Engineering accompanying the Development of iDrive). 3/2002, it - Information Technology, Vol. 44, p. 145. ISSN 1611-2776 (2002)

Bettini, A., et al.: Vision-assisted control for manipulation using virtual fixtures. IEEE Transactions on Robotics, Vol. 20, p. 953-966 (2004)

Donmez, B., et al.: A literature review of distraction mitigation strategies. SAVE-IT Project Deliverable. (2004)

Gibson, J.J.: The theory of affordances. R. Shaw e J. Bransford. Perceiving, acting, and knowing: Toward an ecological psychology. Hillsdale, NJ : Erlbaum, p. 67-82 (1977)

Likert, R.: A technique for the measurement of attitudes. Archives of Psychology, Vol. 140, 55, (1932)

Nolin, J.T., Stemniski, P.M., Okamura A.M.: Activation cues and force scaling methods for virtual fixtures. 11th Symposium on Haptic Interfaces for Virtual Environment and Teleoperator Systems, Chicago, IL, USA (2003)

Nowakowski, C., Utsui, Y. e Green, P.: Navigation system evaluation: The effects of driver workload and input devices on destination entry time and driving performance and their implications to the SAE recommended practice. The University of Michigan Transportation Research Institute.UMTRI-2000-20 (2000)

Payandeh, S., Stanisic, Z.: On application of virtual fixtures as an aid for telemanipulation and training. 2002. Proceedings of the Symposium on Haptic Interfaces for Virtual Environment and Teleoperator Systems (2002) 
Rosenberg, L.: Virtual fixtures: Perceptual tools for telerobotic manipulation. Proceedings of the Virtual Reality Annual International Symposium (1993)

Secchi C., Stramigioli, S., Fantuzzi, C.: Intrinsically passive force scaling in haptic interfaces. Proceedings of IEEE/RSJ International Conference on Intelligent Robots and Systems. Beijing, China (2006)

Young, K., Regan, M. e Hammer, M.: Driver distraction: a review of the literature. Monash University Accidents Research Centre. Report 206. (2003)

Wang, D., et al.: Haptic overlay device for flat panel touch displays. Proceedings of the Symposium on Haptic Interfaces for Virtual Environment and Teleoperator Systems. Chicago, Illinois, USA (2004) 